\title{
Clinical and pathological features of women with adnexal masses admitted as emergency cases to the Gynaecology Department of West Kazakhstan University
}

\author{
Akmaral S. Adilgereyeva ${ }^{1}$, Ibrahim A. Abdelazim ${ }^{2,3}$, Gulmira A. Zhurabekova ${ }^{1}$ \\ ${ }^{1}$ Department of Normal and Topographical Anatomy, West Kazakhstan Marat Ospanov Medical University, Aktobe, Kazakhstan \\ ${ }^{2}$ Department of Obstetrics and Gynecology, Ain Shams University, Cairo, Egypt \\ ${ }^{3}$ Department of Obstetrics and Gynecology, Ahmadi Hospital, Kuwait Oil Company, Ahmadi, Kuwait
}

\begin{abstract}
Aim of the study: To detect the clinical and pathological features of women with adnexal masses (AMs) admitted as emergency cases to the Gynaecology Department of West Kazakhstan University.

Material and methods: A retrospective analysis of the data of women with AMs admitted as an emergency cases to the Gynaecology Department of West Kazakhstan University. The collected data include: age, age of menarche and age of menopause, presenting symptoms, admission criteria - either self-referral or refereed from another department, ultrasound findings, associated pregnancy, associated pathology of the female genital tract, and post-operative histological results of surgically excised AMs (gold standard).

Results: $77.04 \%$ (245/318) of the studied AMs were found in the reproductive age group. The main causes for surgical intervention for the studied AMs was ruptured ovarian cyst in $27.1 \%$ or adnexal torsion in $9.7 \%$. The available histological results of the surgically managed AMs showed the following: functional ovarian cyst in 36.2\% (115/318), benign ovarian neoplasms (BONs) in 18.55\% (59/318), and borderline malignant ovarian tumours in $0.63 \%$ (2/318). $44.34 \%$ of the studied AMs were associated with pregnancy, $49.3 \%$ with chronic tubo-ovarain diseases such as salpingo-oophoritis, $14.8 \%$ with cervical pathology and pelvic inflammatory diseases, $11.3 \%$ with uterine leiomyomas, and $4.4 \%$ with endometrial hyperplasia.

Conclusions: AMs were more common in the reproductive age group $(77.04 \%)$, and $44.34 \%$ of the studied AMs were associated with pregnancy. The main causes of surgical intervention for the studied AMs were ruptured ovarian cyst in $27.1 \%$ or adnexal torsion in $9.7 \%$.
\end{abstract}

Key words: prevalence, clinical, pathological, adnexal masses (AMs), West Kazakhstan.

\section{Introduction}

The incidence of benign ovarian cysts is $14-18 \%$ in postmenopausal women, $7 \%$ in reproductive-age women, and $0.2-5 \%$ during pregnancy [1]. About $2 \%$ of the adnexal masses (AMs) are malignant or borderline malignant [2]. The pre-operative differentiation between benign and malignant AMs is important to decide further clinical and surgical management [3-4].

Benign ovarian neoplasms (BONs) are characterised by the great variation of their morphological structures and clinical manifestations [5]. They are often accompanied by complications that necessitate surgical intervention, such as rupture, torsion, and/or suppuration [5-6]. BONs have an impact on the reproductive potential [5-6]. The available evidence indicates that the risk of torsion when ovarian cysts present during pregnancy is $22 \%$ [7] and this risk increases to $45 \%$ when symptomatic ovarian cysts present [8].

Despite the available evidence, only a few studies have been conducted in Kazakhstan to detect the clinical and pathological features of AMs, possible complications, and their predictors. Therefore, this study was designed to detect the clinical and pathological features of women with AMs admitted as emergency cases to the Gynaecology Department of West Kazakhstan University.

\section{Material and methods}

A retrospective analysis of the data of women with AMs admitted as emergency cases to the Gynaecology Department of West Kazakhstan University from Janu- 
ary 2016 to January 2018 after approval of the Institute Ethical Committee.

The collected data included the following: 1) age, age of menarche, and age of menopause; 2) admission criteria; either self-referral (patient presented to the emergency department due to abdominal pain) or referred from another department (surgical department after exclusion of surgical causes of abdominal pain); 3) pre-operative findings on gynaecological examination and on pelvic ultrasound examination; 4) associated pregnancy (intrauterine or extrauterine [ectopic pregnancy] and/or miscarriage); 5) associated concomitant pathology of the female genital tract beside the adnexal mass (uterine leiomyomas and/or genital infection, such as pelvic inflammatory disease [PID]); 6 ) the results of the histopathological examination of the removed AMs (gold standard).

Collated clinical data included the following: medical history, physical examination on admission, and laboratory investigations (complete blood count, total leucocytic count [TLC] and human chorionic gonadotropins [ $\beta$-hCG], and ultrasound scan findings). Collected ultrasound findings included the following: the size and consistency of the mass (cystic, solid, or mixed), whether the examined mass was unilateral or bilateral, the presence or absence of septations, mural nodules, papillary projection, and free fluid in the pelvis [9]. The presence of papillary projection, ascites, and mural nodules within the adnexal mass raise the suspicion of malignancy, especially in premenopausal or postmenopausal women, whereas the absence of these findings suggests a benign diagnosis [9].

The conservative or surgical management decision was based on the presence or absence of abdominal or pelvic symptoms after admission and suspicion of tubo-ovarain abscess (lower abdominal pain, adnexal tenderness associated with fever $\left(>38^{\circ} \mathrm{C}\right)$, elevated TLC, cervical motion tenderness, laboratory documentation of cervical infection with $N$. gonorrhoeae and/ or C. trachomatis), ectopic pregnancies (quantitative $\beta$-hCG with adnexal mass and empty uterus), and suspected torsion or rupture of the adnexal mass [9].

\section{Statistical analysis}

Collected data were tabulated and statistically analysed using SPSS (Statistical Package for the Social Science, version 22; IBM Corp, New York, USA) for Microsoft Windows. Data are presented as mean \pm standard deviation (SD), number, and percentage. The $\chi^{2}$ test was used for statistical analysis of the qualitative variables, and Student's $t$-test was used for statistical analysis of quantitative variables. The primary outcome measures the clinical and pathological features of women with AMs admitted as emergency cases to the Gynaecology Department of West Kazakhstan
University. The $p$-values $<0.05$ were considered statistically significant.

\section{Results}

During the study period (January 2016 to January 2018), 318 women with provisional diagnosis of adnexal mass were admitted as emergency cases to the Gynaecology Department of West Kazakhstan University. The mean age of the studied women was $33.79 \pm 9.33$ years (range 18-62 years). There was no significant difference between the development of the adnexal mass and the age of menarche $(p=0.2)$ and/or the age of menopause $(p=0.2)$.

Regarding the causes of admission, $94.7 \%$ (301/318) of the studied women with AMs were admitted due to abdominal pain (91.4\% [275/301] admitted directly from the Emergency Room to the Gynaecology Department and 8.6\% (26/301) referred from the Surgical Department after exclusion of surgical causes of abdominal pain), and 5.3\% (17/318) were admitted following heavy menstrual bleeding (menorrhagia) and AMs discovered during work-up for menorrhagia.

Regarding the origin of the AMs, 55\% (175/318) originated from the right adnexa, 41.2\% (131/318) from the left adnexa, and 3.8\% (12/318) were of bilateral origin (Table 1).

The studied women classified according to their ages, as follows: group I (18-40 years), group II (41-44 years), group III (45-49 years), and group IV (> 50 years). The prevalence of AMs according to the age of the studied groups was as follows: $77.04 \%$ (245/318) in group I, $8.81 \%(28 / 318)$ in group II, $7.55 \%(24 / 318)$ in group III, and $6.6 \%(21 / 318)$ in group IV (Table 1$)$.

The ultrasound provisional diagnosis was benign adnexal mass (BAM) in $80.4 \%$ and ovarian cyst in $19.6 \%$ of group I, $64.3 \%$ and $35.7 \%$, respectively, in group II, $70.8 \%$ and $29.2 \%$, respectively, in group III, and $66.6 \%$ and $33.4 \%$, respectively, in group IV. One hundred and seventy-six (176/318 [55.35\%]) women managed surgically (36.17\% [115/318] laparoscopically and 19.18\% [61/318] by laparotomy), while $44.65 \%(142 / 318)$ of the cases were managed conservatively (Table 1 ). The main causes for the surgical intervention for the studied AMs was acute abdominal pain due to ruptured ovarian cyst in $27.1 \%$ or adnexal torsion in $9.7 \%$.

The available histological examination of the surgically managed AMs showed the following: functional ovarian cyst in $36.2 \%$ (115/318), BONs in $18.55 \%$ (59/318), and borderline malignant ovarian tumours in $0.63 \%(2 / 318)$ in group II and IV (Table 1$)$. Regarding the association of the studied AMs with pregnancy and other pathological conditions of the female genital tract; $44.34 \%$ of the studied AMs were associated with pregnancy and 49.3\% (157/318) were associated with other pathological conditions of the female genital tract. 
Table 1. Clinical and pathological features of the studied adnexal masses (AMs) $(n=318)$

\begin{tabular}{|c|c|}
\hline Variable & $\begin{array}{c}\text { Studied } \\
\text { women } \\
n(\%)\end{array}$ \\
\hline \multicolumn{2}{|l|}{ Prevalence of the studied AMs according to the age } \\
\hline Group I (18-40 years) & $245(77.04)$ \\
\hline Group II (41-44 years) & $28(8.81)$ \\
\hline Group III (45-49 years) & $24(7.55)$ \\
\hline Group IV (> 50 years) & $21(6.6)$ \\
\hline \multicolumn{2}{|l|}{ Origin of the studied AMs } \\
\hline Right adnexa & $175(55)$ \\
\hline Left adnexa & $131(41.2)$ \\
\hline Bilateral & $12(3.8)$ \\
\hline \multicolumn{2}{|l|}{ Management of the studied AMs } \\
\hline Conservative management & $142(44.65)$ \\
\hline Surgical management & $176(55.35)$ \\
\hline laparoscopic & $115(36.17)$ \\
\hline laparotomy & $61(19.18)$ \\
\hline \multicolumn{2}{|l|}{ Histological results of the surgical managed AMs } \\
\hline Functional ovarian cyst & $115(36.2)$ \\
\hline BONs & $59(18.55)$ \\
\hline Borderline malignancy & $2(0.63)$ \\
\hline \multicolumn{2}{|l|}{$\begin{array}{l}\text { Association of the studied AMs with pregnancy } \\
\text { and the outcome of pregnancy }\end{array}$} \\
\hline Spontaneous miscarriage & $56(17.61)$ \\
\hline Ectopic pregnancy & $33(10.38)$ \\
\hline Full term pregnancy & $32(10.06)$ \\
\hline Non-viable preterm pregnancy ( $<24$ weeks) & $20(6.29)$ \\
\hline \multicolumn{2}{|l|}{$\begin{array}{l}\text { Association of the studied AMs with other } \\
\text { pathological conditions of the female genital tract }\end{array}$} \\
\hline Salpingo-oophoritis & $157(49.3)$ \\
\hline Cervical pathology and PID & $47(14.8)$ \\
\hline Uterine leiomyoma & $36(11.3)$ \\
\hline Endometrial hyperplasia & $14(4.4)$ \\
\hline
\end{tabular}

The pregnancy outcome and the other associated pathological conditions of the female genital tract are shown in Table 1 and Figure 1.

\section{Discussion}

Three hundred and eighteen women included in this retrospective study to detect the clinical and pathological features of women with AMs admitted as emergency cases to the Gynaecology Department of West Kazakhstan University. In this study, although there was no significant difference between the development of AM and the age of menarche and/or the age of menopause, $77.04 \%(245 / 318)$ of the studied AMs were found in group I (reproductive age group [18-40 years]). Similarly, Taş and Yavuz found that $91 \%$ of their studied BONs were complicated with adnexal torsion in the reproductive age group [5]. In addition; Huang et al. and Robertson et al. concluded that most of the ovarian or adnexal torsion occurs in the reproductive age, and the presence of ovarian cyst or mass $>5 \mathrm{~cm}$ predisposes to ovarian torsion [10, 11].

This study found that $49.3 \%$ (157/318) of the studied AMs were associated with chronic tubo-ovarain diseases such as salpingo-oophoritis, $14.8 \%$ (47/318) with cervical pathology and pelvic inflammatory diseases (PID), 11.3\% (36/318) with uterine leiomyomas, and $4.4 \%(14 / 318)$ with endometrial hyperplasia. In addition, $44.34 \%$ of the studied AMs were associated with pregnancy $(17.61 \%$ of the pregnancies ended with miscarriage, in $10.38 \%$ the outcome of pregnancy was ectopic, in $10.06 \%$ the pregnancy outcome was fullterm delivery, and in $6.29 \%$ the pregnancy ended with non-viable preterm delivery $<24$ weeks' gestation).

Mimoun et al. found that the incidence of ovarian cyst is about 0.2-5\% during pregnancy [1]. de Haan et al. found that AMs during pregnancy are not uncommon, with $0.2-2 \%$ incidence, depending on the stage of pregnancy [12]. In addition, they concluded that ovarian cysts during pregnancy should be carefully evaluated

Association of the studied AMs with pregnancy and other pathological conditions of the female genital tract

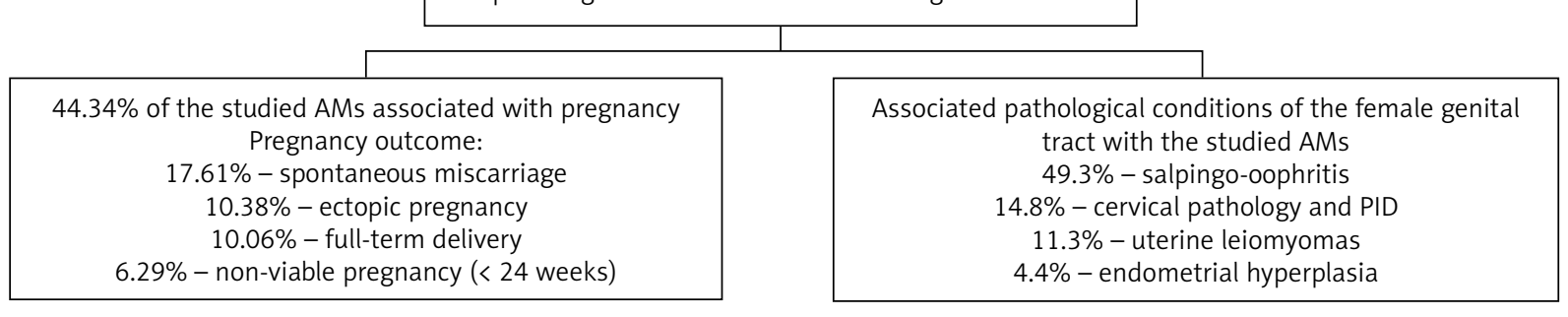

PID - pelvic inflammatory diseases

Fig. 1. The pregnancy outcome and the associated pathological conditions of the female genital tract with the studied adnexal masses (AMs) 
to identify women who need surgical interventions and those who can managed conservatively [12].

One hundred and seventy-six (176/318 [55.35\%]) of the studied women with adnexal mass were managed surgically: $36.17 \%$ (115/318) laparoscopically and $19.18 \%(61 / 318)$ by laparotomy, while $44.65 \%$ (142/318) of the cases were managed conservatively. Similarly, Taş and Yavuz found that in the BONs with adnexal torsion, radial surgical treatment and laparotomy were considered in $54.6 \%$ of the studied cases, while conservative treatment was considered in 45.4\% [5]. Taş and Yavuz concluded that laparoscopy is a well-defined surgical approach and widely used by surgeons to salvage the ovarian tissue in cases of BONs [5].

The main causes for the surgical intervention for the studied AMs were acute abdominal pain due to ruptured ovarian cyst in $27.1 \%$ or adnexal torsion in $9.7 \%$. Taş and Yavuz found that the main cause of surgical intervention in BONs was adnexal torsion (AT) in 65.1\% of patients and ovarian cyst rupture (OCR) in $31 \%$ of patients [5]. In addition, Sasaki and Miller concluded that the AT is the fifth most common gynaecological emergency, and when AT was suspected, timely diagnostic laparoscopy was indicated to preserve ovarian function and future fertility [13].

The available histological examination of the surgical managed AMs showed the following: functional ovarian cyst in $36.2 \%$ (115/318), BONs in 18.55\% (59/318), and borderline malignant ovarian tumours in $0.63 \%(2 / 318)$ in group II (41-44 years) and IV (> 50 years). Previous studies concluded that the risk of malignancy in women with AMs increased in postmenopausal women, especially with the presence of solid components, bilaterality based on ultrasound findings and high CA-125 [14, 15]. In addition, the guidelines of the American college of Obstetricians and Gynaecologists (ACOG) mentioned that the presence of papillary projection, ascites, and mural nodules within the adnexal mass raises the suspicion of malignancy, especially in premenopausal or postmenopausal women, whereas the absence of these findings suggests a benign diagnosis [9].

To the best of our knowledge, this study was the first to be conducted in West Kazakhstan University, to detect the clinical and pathological features of women with AMs admitted as emergency cases. The retrospective nature of the study and the non-available detailed data regarding clinical evaluation, ultrasound findings on admission, and histological results of all studied women were limitations of this study. The pre-operative discrimination between benign and malignant AMs is important to decide further management. Therefore, another prospective study is ongoing to correlate the pre-operative risk malignancy index (RMI) with the post-operative histological results (gold standard) of the surgical managed AMs.

\section{Conclusions}

AMs were more common in the reproductive age group (77.04\%), and $44.34 \%$ of the studied AMs were associated with pregnancy. The main causes for surgical intervention for the studied AMs were ruptured ovarian cyst in $27.1 \%$ and adnexal torsion in $9.7 \%$.

\section{Acknowledgments}

The authors are grateful for the women who agreed and gave consent to be included in this study.

\section{Disclosure}

The authors report no conflict of interest.

\section{References}

1. Mimoun C, Fritel X, Fauconnier A, et al. [Epidemiology of presumed benign ovarian tumors]. J Gynecol Obstet Biol Reprod (Paris) 2013; 42: 722-729.

2. Marret $\mathrm{H}$, Cayrol M. [Sonographic diagnosis of presumed benign ovarian tumors]. J Gynecol Obstet Biol Reprod (Paris) 2013; 42: 730-743.

3. Javdekar R, Maitra N. Risk of Malignancy Index (RMI) in Evaluation of Adnexal Mass. J Obstet Gynaecol India 2015; 65: 117-121.

4. Sönmezer M, Taşkın S. Fertility preservation in women with ovarian endometriosis. Womens Health (Lond) 2015; 11: 625-631.

5. Taş E, Yavuz A. Adnexal Torsion: Difficulty in Diagnosis and Management. Med Bull Haseki 2018; 56: 187-191.

6. Duigenan S, Oliva E, Lee SI. Ovarian torsion: diagnostic features on CT and MRI with pathologic correlation. Am J Roentgenol 2012; 198 : W122-131.

7. Yen CF, Lin SL, Murk W, et al. Risk analysis of torsion and malignancy for adnexal masses during pregnancy. Fertil Steril 2009; 91: 1895-1902.

8. Mavromatidis G, Sotiriadis A, Dinas K, et al. Large luteinized follicular cyst of pregnancy. Ultrasound Obstet Gynecol 2010; 36: 517-520.

9. American College of Obstetricians and Gynecologists. ACOG Practice Bulletin. Management of adnexal masses. Obstet Gynecol 2007; 110: 201-214.

10. Huang C, Hong MK, Ding DC. A review of ovary torsion. Ci Ji Yi Xue Za Zhi 2017; 29: 143-147.

11. Robertson JJ, Long B, Koyfman A. Myths in the evaluation and management of ovarian torsion. J Emerg Med 2017; 52: 449-456.

12. de Haan J, Verheecke M, Amant F. Management of ovarian cysts and cancer in pregnancy. Facts Views Vis Obgyn 2015; 7: 25-31.

13. Sasaki KJ, Miller CE. Adnexal torsion: review of the literature. J Minim Invasive Gynecol 2014; 21: 196-202.

14. Karakaya BK, Ozgu E, Kansu HC, et al. Evaluation of Probably Benign Adnexal Masses in Postmenopausal Women. Rev Bras Ginecol Obstet 2017; 39: 229-234

15. Dora SK, Dandapat AB, Pande B, Hota JP. A prospective study to evaluate the risk malignancy index and its diagnostic implication in patients with suspected ovarian mass. J Ovarian Res 2017; 10: 55. 